\title{
Les firmes agroalimentaires occidentales à la conquête de l'Europe centraleLes industriels laitiers en Pologne, Slovaquie et République Tchèque
}

Occidental food-processing firms at the conquest of Central EuropeMilk industrialists in Poland, Slovakia and Czech Republik

Agro-industrielle Unternehmen in MitteleuropaMilchwirtschaft in Polen, Slowakei und der tschechischen Republik

\section{Daniel Ricard}

\section{OpenEdition Journals}

Édition électronique

URL : http://journals.openedition.org/rge/3087

DOI : 10.4000/rge.3087

ISSN : 2108-6478

Éditeur

Association des géographes de l'Est

Édition imprimée

Date de publication : 31 décembre 2010

ISSN : 0035-3213

Référence électronique

Daniel Ricard, «Les firmes agroalimentaires occidentales à la conquête de l'Europe centraleLes industriels laitiers en Pologne, Slovaquie et République Tchèque ", Revue Géographique de l'Est [En ligne], vol. 50 / 3-4 | 2010, mis en ligne le 17 octobre 2011, consulté le 10 décembre 2020. URL : http:// journals.openedition.org/rge/3087 ; DOI : https://doi.org/10.4000/rge.3087

Ce document a été généré automatiquement le 10 décembre 2020.

Tous droits réservés 


\title{
Les firmes agroalimentaires
} occidentales à la conquête de l'Europe centraleLes industriels laitiers en Pologne, Slovaquie et République Tchèque

\author{
Occidental food-processing firms at the conquest of Central EuropeMilk \\ industrialists in Poland, Slovakia and Czech Republik \\ Agro-industrielle Unternehmen in MitteleuropaMilchwirtschaft in Polen, \\ Slowakei und der tschechischen Republik
}

Daniel Ricard

\section{Introduction}

1 Les profondes transformations ayant eu lieu en Europe centrale après 1989 ont largement renouvelé le champ de recherche propre à cette région. En géographie rurale, plusieurs thèses ont ainsi été soutenues (G. Zrinscak, 1997; G. Lacquement, 2002 ; J. Maciulyte, 2001; B. Von Hirschhausen, 1997), mettant l'accent sur les évolutions complexes du foncier et des exploitations agricoles, puis s'intéressant davantage aux processus de développement à l'œuvre dans ces campagnes (M. Lompech, 2003). Sur place, on s'est aussi intéressé à ces thèmes, comme à la production agricole elle-même (P. Spisšiak en Slovaquie...). La question de la transformation des produits agricoles, sans être oubliée, a toutefois été assez peu abordée par les chercheurs, même si on doit citer à ce propos G. Zrinscak ou B. Domańsky (Cracovie) ainsi que l'ancienne revue « Le Courier des Pays de l'Est ».

Cet article aborde justement le thème particulièrement mouvant de l'agroalimentaire, à travers l'exemple du secteur laitier. Il s'appuie sur la compilation de la presse 
professionnelle laitière (française essentiellement) ainsi que sur de nombreuses enquêtes de terrain effectuées depuis 1999 en Pologne, République Tchèque et surtout Slovaquie.

\section{I - Le contexte général : une profonde réorganisation de l'économie laitière}

3 Après 1989, l'industrie agroalimentaire des anciennes démocraties populaires s'est profondément réorganisée. Le passage à l'économie de marché s'y est accompagné d'une privatisation généralisée des outils industriels, d'une sélection des usines et d'une nette amélioration de la productivité des entreprises. L'industrie laitière a pleinement participé à ce mouvement, marqué par ailleurs par l'arrivée massive de capitaux étrangers, surtout ouest européens.

4 Même si le changement de système économique et politique a été rapide, le processus s'apparente toutefois à une transition, relativement progressive, d'un système vers un autre. Dès lors, la part des héritages historiques est importante, les évolutions étant par ailleurs inégales d'un pays à l'autre en fonction des politiques gouvernementales et de l'attractivité nouvelle de chaque Etat.

\section{A - Le poids des héritages}

En dépit de nombreuses lacunes structurelles, Pologne et Tchécoslovaquie sont bien, en 1989, des territoires économiquement avancés, même si les différences sont nombreuses d'un pays à l'autre, voire d'une région à l'autre. A l'amont, les deux Etats produisent respectivement 15,41 et 7,10 milliards de litres en 1990 (l'équivalent de $85 \%$ de la production française). A l'aval, l'Industrie Agro-Alimentaire (IAA), dont les racines sont très anciennes - pensons aux sucreries et brasseries tchèques (Zrinscak, 1997) - a été modernisée par le régime socialiste dès les années 1950 et s'avère assez performante. Certes, ces usines manquent de technologie et l'on n'atteint ni la technicité, ni le gigantisme, ni les performances des laiteries des rivages de la mer du Nord, mais l'outil de transformation reste globalement efficace. $40 \%$ des usines tchécoslovaques ainsi que les seize plus grosses coopératives polonaises collectent alors plus de 100 millions de litres de lait (ONILAIT, 1992).

Cette industrie se caractérise par une organisation très centralisée, en conformité avec la pensée marxiste. Cela est particulièrement vrai en Tchécoslovaquie; les campagnes polonaises, moins systématiquement collectivisées et demeurées plus paysannes, se prêtant moins à ce type d'organisation pyramidale. Prague avait ainsi défini 11 grandes régions laitières, plus celle de la capitale, administrées par des Directions centrales et contrôlées par le Ministère de l'agriculture (fig. 1). 


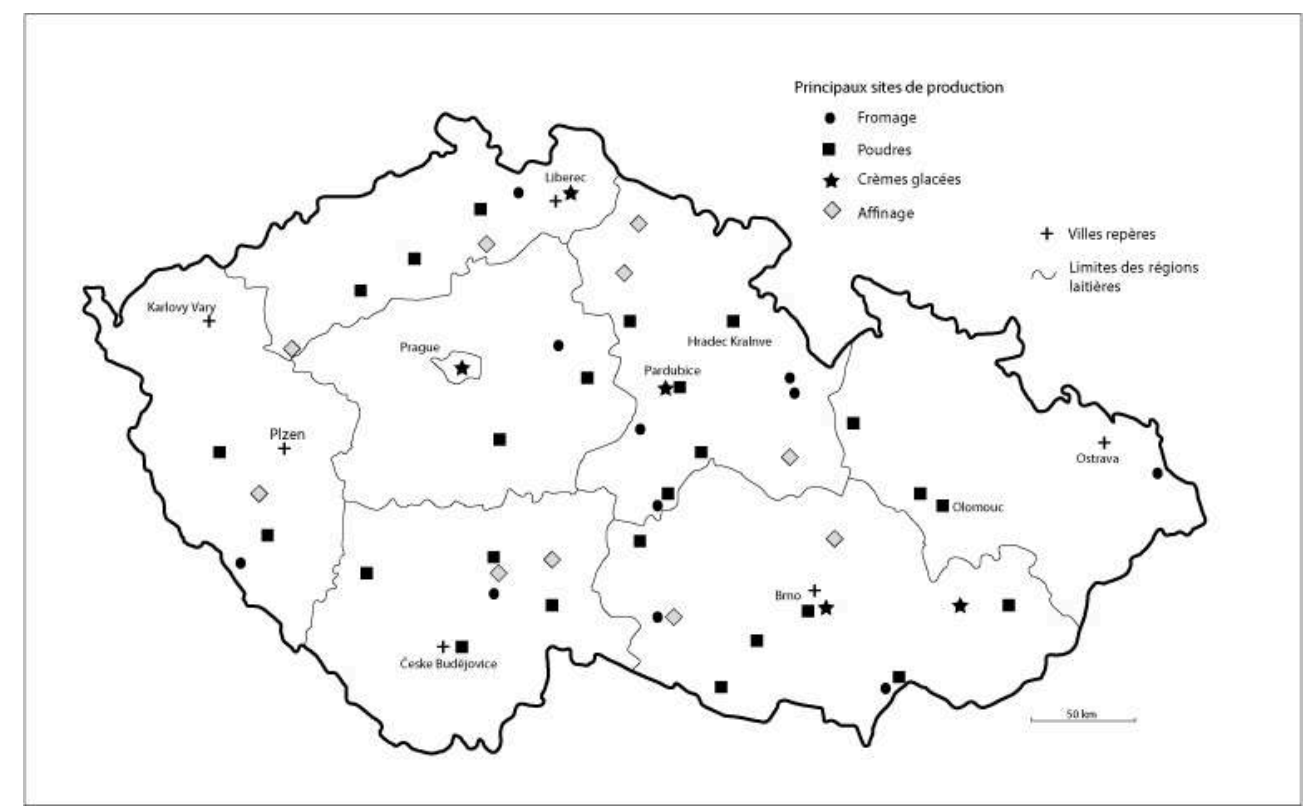

Source : D. Ricard, d'après VŠCHT, Českomoravský Svaz Mlékárenský et G. Zrinscak, (d'après A. Gôtz)

7 Cette centralisation se double d'une forte volonté d'autonomie des territoires. On s'efforce alors de tout produire ou presque dans chaque région laitière (lait de consommation, yaourts, fromages, beurre, poudres...), même si certaines productions spécifiques n'ont qu'un site dédié (cas de Zábřeh na Moravě pour le lait infantile). La diversification se retrouve au sein même des usines quand celles de l'Ouest du continent sont, dès cette époque, nettement plus spécialisées.

\section{B - Les IAA à l'épreuve de la transition}

8 La chute des régimes marxistes bouleverse ces IAA, sans toutefois conduire à leur effondrement. Il faut dire que ces deux (puis trois) pays connaissent une transition relativement paisible qui n'a rien de commun avec ce que l'on observe dans l'ex-URSS ou les Balkans. La démocratie se met en place, le cadre juridique nouveau s'installe assez vite, les services publics fonctionnent, les infrastructures aussi et l'ouverture vers l'Ouest est rapide. Dans ce contexte, qui est bien toutefois celui de bouleversements majeurs, on assiste à de nombreuses évolutions (fig. 2).

91 : On constate tout d'abord, en haut lieu comme au sein de la société, une évidente volonté de favoriser l'initiative privée. C'est surtout le cas en Slovaquie où se créent des dizaines de petites laiteries, hélas souvent peu viables (Carlier, 2001). En Pologne, où l'initiative privée avait résisté, on retrouve le même mouvement. Quant aux paysans des Carpates, ils développent depuis longtemps diverses formes de vente directe, à la ferme, sur les marchés... ou dans la rue principale de la station de Zakopane. Le mouvement touche en revanche beaucoup moins la Tchéquie.

2: On observe dans le même temps une crise généralisée des IAA (Carlier, 2000). La filière laitière reflète alors parfaitement les difficultés économiques de l'instant, prise en tenaille entre la baisse drastique de la consommation (-20\% environ, en liaison avec le fort recul du pouvoir d'achat) et un sévère repli de la production suite à la 
déstructuration des fermes collectives. Résultat, de 1989 à 1992, la collecte baisse de 41 $\%$, en Pologne comme en Tchécoslovaquie (Tab. 1).

11 Cette crise générale, stimule une forme de sélection naturelle des IAA. Les plus vétustes ne sont pas toujours les plus fragiles car elles ont l'avantage d'être amorties. D'autres, qui avaient récemment investi, souffrent à l'inverse d'un trop lourd endettement. Mais il faut aussi prendre en compte d'autres facteurs. Les affaires les plus pénalisées sont surtout celles qui sont orientées vers des créneaux peu porteurs, ou qui dépendent de marchés à l'export qui se ferment rapidement (fromages à destination des pays arabes par exemple), celles encore qui sont les plus mal gérées, celles enfin qui sont victimes de malversations.

Figure 2 : L'évolution de la collecte de lait en Pologne et en Tchécoslovaquie (en millions de litres)

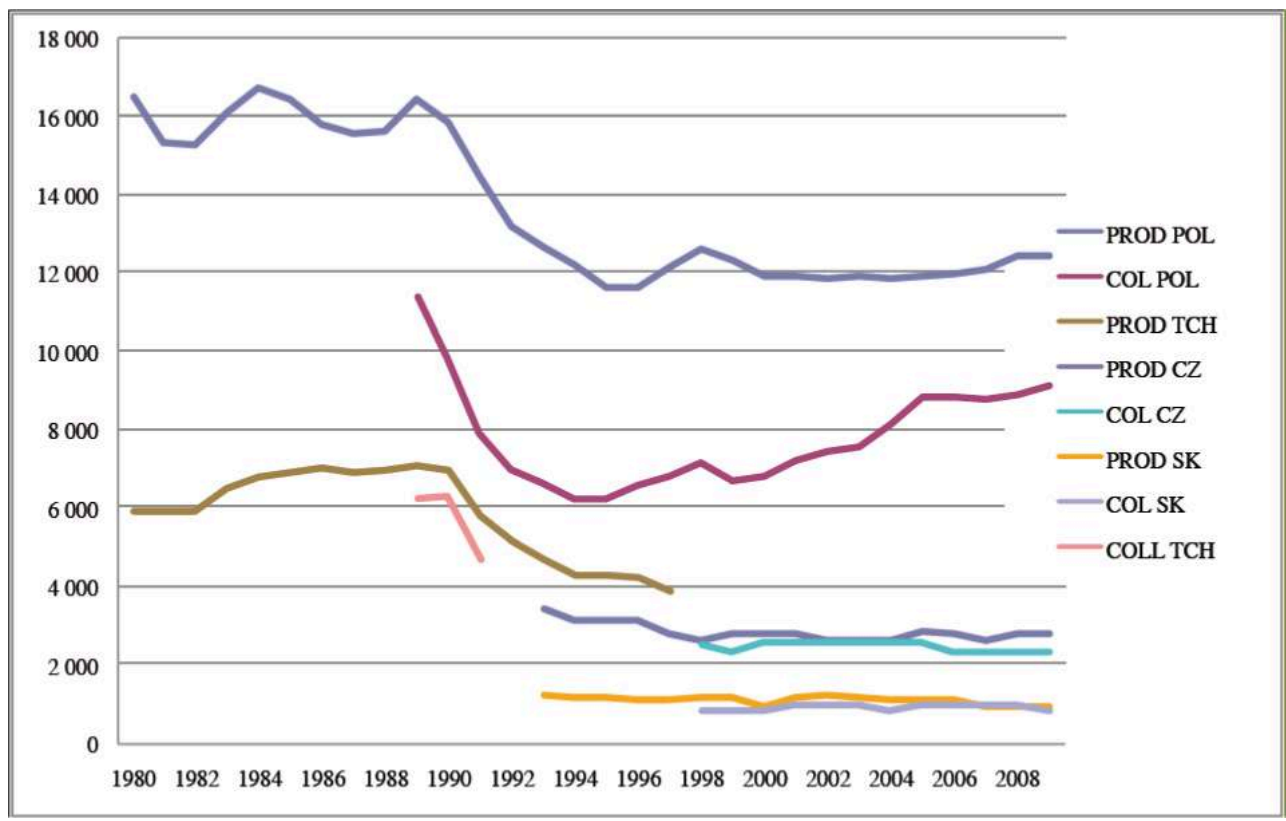

Source : ONILAIT, CNIEL, FAO

123 : L'évolution des filières laitières, comme celle de toutes les IAA, doit aussi beaucoup à l'action des gouvernements, qui contrôlent plus ou moins bien l'économie, autorisent ou non la privatisation des laiteries, en maîtrisent le calendrier et favorisent ou non l'arrivée des capitaux étrangers. Les flux d'Investissements Directs Etrangers (IDE) dépendent en effet largement de la législation et de l'environnement politique. En Pologne comme en Tchéquie, ce dernier est globalement satisfaisant et les investisseurs affluent. En Slovaquie, en revanche, V. Méčiar adopte un certain repli nationaliste et il faut attendre la victoire de la coalition conduite par le libéral M. Dzurinda (1998) pour que le pays s'ouvre vraiment.

\section{II - Les laiteries occidentales à la « conquête de l'Est »}

Les IDE se développent rapidement à partir du milieu des années 1990 et le secteur laitier y prend une part significative. Les "proies » sont les entreprises qui, suite aux grandes lois de privatisation, sont souvent vendues selon un processus inégalement rapide d'un pays à l'autre, plus tardif en Slovaquie. Tout l'art est alors, pour les 
«prédateurs », de repérer les entreprises les plus intéressantes, celles qui sont dotées du meilleur potentiel. Ces investisseurs agissent de manière complexe, fruit d'une somme d'initiatives individuelles plutôt que résultat d'une attitude collective. Quelques tendances générales se dessinent toutefois.

Premier élément, on privilégie la reprise d'unités existantes à la création d'usines, en liaison avec des prix de vente assez bas, des potentialités de développement réelles et un coût de la main-d'œuvre susceptible de compenser la faible productivité. Rares sont donc les usines nouvelles, même si l'on peut signaler la construction récente à Zvolen (Slovaquie) d'une unité de produits frais destinés au marché slovaque par le Français Senoble qui y rapatrie les activités de deux laiteries reprises en 2005 et en 2007. Les industriels ont agi différemment en ex-RDA où ils ont davantage construit, notamment la gigantesque usine de Leppersdorf (groupe Müller), qui transforme 1,6 milliard de litres de lait par an! Il faut dire que, dans ces nouveaux länder, l'avantage du coût du travail se réduisit très vite et que l'on put cumuler aides européennes et fédérales.

Autre caractéristique : le profil de ces entreprises. Celles de moins d'une centaine de salariés restent à l'écart du mouvement, sauf exception, et les investissements relèvent, en général, de grands groupes laitiers qui avaient amorcé leur développement à l'international dès les années 1980, voire 1970, et qui trouvent là un accélérateur de croissance. C'est le cas, côté français, de Danone, Bel, Lactalis (Besnier) et Bongrain, tous massivement présents. On rencontre aussi quelques grosses PME régionales, qui arrivent un peu plus tard, comme Senoble, qui mise successivement sur l'Espagne (2002), la Slovaquie (2005), la Grande Bretagne (2007) et l'Italie (2008). Les grosses PME bavaroises entrent dans ce groupe, même si elles ont moins investi dans ces pays. Les coopératives sont, pour leur part, beaucoup moins agressives, pénalisées par des moyens financiers plus faibles, par leur statut, peut-être aussi par la médiocre image de tout ce qui relève du collectif en Europe centrale? Ainsi, SODIAAL investit directement dans la transformation (à Lenzo en Pologne, à Slušovice en Moravie), avant de privilégier la recherche de partenaires franchisés pour diffuser sa marque Yoplait. Quant aux grandes coopératives allemandes, elles sont systématiquement absentes de ces régions.

Sur le plan géographique, les investisseurs sont presque toujours des Européens de l'Ouest, le reste du monde (USA, Japon, Océanie...) étant quasiment absent du débat.

Les Français ont été les investisseurs les plus actifs, notamment en Slovaquie où ils contrôlent approximativement $60 \%$ de la collecte ! Il faut dire que l'industrie laitière française était, dès 1989 , très puissante et pouvait s'appuyer sur des marques qui trouvèrent vite de nombreux clients en Europe centrale. Ainsi, Danone investit dès 1991 à Wola, vers Varsovie, puis à Benešov, en Tchéquie, en 1992. Le plus actif est incontestablement le fromager Bongrain qui s'installe très tôt en Pologne (en 1993 à Skierniewice, près de Varsovie, puis à Pası:Ek, près de Gdansk) et en Tchéquie (Pribina à Pribyslav) et fait de l'Europe centrale une priorité stratégique. Il s'installe en Slovaquie en 2000 / 2001 en reprenant la grosse laiterie Liptov de Liptovský Mikulaš, puis celles de Zvolen (Wittmann a Syn) et de Nové Mesto nad Vahom (Millex). Dès 2002, le groupe exploite 18 usines dans les PECO et y emploie 4200 salariés (RLF, 2002)! Le leader européen Lactalis, qui avait pourtant acquis le Polonais Polser en 1996, attendra dix ans avant de vraiment passer à l'offensive. Il revient dans ce pays en 2006 (en reprenant la coopérative de Kurov) puis s'implante avec force en République Tchèque (groupe Promil de Novy Bydžov, Mlekarna Klatovy et Moravskoslezke Mlekarna à Kunin en 
2007), mais reste absent de Slovaquie. En fait, sa priorité stratégique est autant en exURSS, où il investit en Ukraine (Nikolaïev), en Russie, en Moldavie et au Kazakhstan. Quant à Entremont, il est présent en Tchéquie (Mlekarna Stř́bro, 2001) et en Pologne (WĘgrów) via sa filiale Eurosérum. Enfin, les Fromageries Bel ont repris des fabriques de fromage fondu (Zeletavska Syrarna près de Telč en 2000, Zemplinska Mliekareň à Michalovce en 2002), mais aussi des fromageries (Chorzele en 1999, Tvrdošin en 2005, Praska en 2004 et Jaromericka Mlekarna en 2008, une affaire qui s'appuie sur les deux usines de Moravské Budějovice et de Jaroméřice nad Rokytnou), voire des marques (rachat de Gervais à Danone en 2007, de Syrokrem en Slovaquie en 2003).

Bien que voisins, les Allemands ont finalement assez peu investi ici, privilégiant les nouveaux länder, plus faciles à conquérir pour eux et où la manne des fonds européens, arrivée plus tôt, se combinait avec les aides généreuses de Bonn. En outre, l'Allemagne du Nord est alors concentrée sur la restructuration de sa propre filière, autour des gigantesques coopératives Nordmilch et Humana, spécialisées dans les produits industriels standardisés (poudre de lait, emmental, édam...) qui exigent de gigantesques usines associées à des bassins de collecte très denses, un modèle difficile à développer en Europe centrale où les volumes de lait disponibles restent assez limités. Les investissements allemands sont, en fait, venus des grosses laiteries privées bavaroises, Hochland, Meggle (qui contrôle Rajo, la plus grosse laiterie slovaque), Müller et Ehrmann (Tab. 1). Notons toutefois que ces PME se sont au moins autant intéressées à l'Europe balkanique et à la Russie qu'à la Pologne, la Slovaquie et la Tchéquie.

Tableau 1 : Les entreprises bavaroises en Europe centrale et orientale

\begin{tabular}{|c|c|c|}
\hline Entreprises & Caractéristiques & Implantations en Europe centrale et orientale \\
\hline $\begin{array}{l}\text { Müller } \\
\text { (Aretsried) }\end{array}$ & $\begin{array}{l}\text { CA : } 1,77 \text { milliard } € \\
4560 \text { salariés }\end{array}$ & ${ }^{*}$ Mlékarna Pragolaktos Prague ( $50 \%$ en $2005,76 \%$ en 2008) \\
\hline $\begin{array}{l}\text { Hochland } \\
\text { (Heimenkirch) }\end{array}$ & $\begin{array}{l}\text { CA : } 1,08 \text { milliard } \\
4305 \text { salariés }\end{array}$ & $\begin{array}{l}\text { * Pologne : Każmierz (1993), Baranowo (2000, fromages), } \\
\text { Węgrów (2002, fromages) } \\
\text { * Roumanie : Sighişoara (1998, fondu), Sovata (1999, fromage) } \\
\text { * Russie : Raos (Moscou, } 2 \text { usines fromage fondu, } 2000 \text { et 2003) }\end{array}$ \\
\hline $\begin{array}{l}\text { Meggle } \\
\text { (Wasserburg) }\end{array}$ & $\begin{array}{l}\text { CA: } 0,56 \text { milliard. } \\
\text { Collecte: } 180 \mathrm{M} \mathrm{kg}+ \\
250 \text { à l'étranger } \\
1600 \text { salariés }\end{array}$ & $\begin{array}{l}\text { * Slovaquie : Bratislava ( } 2001 / 03,150 \text { millions litres, } \\
300 \text { salariés, yaourts, UHT, crème) } \\
\text { * Croatie : Osijek } \\
\text { * Bosnie : Bilhac } \\
\text { * Albanie : Rhogozne }\end{array}$ \\
\hline $\begin{array}{l}\text { Ehrmann } \\
\text { (Obershönegg) }\end{array}$ & $\begin{array}{l}\text { CA }: 0,65 \text { milliard } \\
1500 \text { salariés }\end{array}$ & $\begin{array}{l}\text { * Ramenskoyé / Moscou (2000, yaourts, crème) } \\
\text { * Střibro (Tchéquie, Velké Meziř́č́i 2001, } 52 \text { millions de litres, } 130 \\
\text { salariés) }\end{array}$ \\
\hline
\end{tabular}

Sources : Revue Laitière Française, sites Internet des entreprises.

Les autres pays ont été moins actifs dans la région, pour des raisons diverses. Solé (Italie) avait beaucoup investi en Slovaquie centrale (Nové Zamky, Trnava, Krupina), 
avant de se retirer aussi vite qu'il était venu, en 2005, suite à la déconfiture de la Parmalat, sa maison mère. La famille Brazzele a été plus sérieuse, avec son investissement de Litove', près d'Olomouc: Orrero fabrique 3000 tonnes de fromages par an et se développe fortement.

Les Autrichiens, pourtant proches par la géographie comme par l'histoire, ont d'abord dû gérer la libéralisation de leur secteur laitier en liaison avec leur adhésion à l'UE et ont assez peu investi ici. Schärdinger acheta dès 1992 la Laiterie Centrale de Bratislava (150 millions de litres par an) avant de la revendre à Meggle. Artax Group possède, de son côté, les deux usines slovaques Milsy de Bánovce nad Bébravou et de Priévidza. Il s'agit en fait d'une entreprise allemande contrôlée par des investisseurs autrichiens depuis 1993.

Pour les Scandinaves, la priorité a bien plus été la réussite de la fusion entre les coopératives Arla (Suède) et MD Foods (Danemark), ainsi que les investissements de cette dernière en Grande-Bretagne. Quant aux grandes coopératives hollandaises Friesland et Campina Melkunie (aujourd'hui fusionnées), elles investirent en Pologne (à Toruń, auprès du conglomérat Elektrim) et en Slovaquie (Strážske, Nitra, Žilina), avant de se retirer en 2006. Enfin, Anglais, Espagnols, Belges et Irlandais sont absents, de même que la grande coopérative finlandaise Valio et que le Suisse Nestlé, même si ses marques sont omniprésentes en grande distribution.

Figure 3 : Les sociétés laitières occidentales en Tchéquie, Slovaquie et Pologne






\section{III - Les investisseurs occidentaux en action en Europe centrale}

L'arrivée massive des investisseurs étrangers bouleverse la transformation laitière. A la différence toutefois d'autres contrées plus orientales, ils peuvent s'appuyer ici sur une production laitière organisée, des usines qui fonctionnent, des marques, des réseaux de distribution qui se modernisent rapidement et enfin sur des marchés qui se développent à partir de 1992/93. Tout sera donc plus facile qu'en Roumanie, en Serbie ou en Ukraine, ce qui n'empêche pas les investisseurs de se retrouver confrontés à de réelles difficultés, industrielles, commerciales et organisationnelles.

\section{A - Réorganiser l'outil industriel}

Les étrangers ont rapidement acquis une place décisive dans ces trois pays, au point d'y contrôler plus de la moitié de la collecte. Ils y détiennent souvent les meilleures entreprises, sont surtout implantées dans les meilleures zones laitières (Moravie, plaine du Danube...) et ont repris nombre de belles marques. Il n'en demeure pas moins que leurs investissements ne constituent pas un long fleuve tranquille. Les difficultés sont de nature différente, mais quelques-unes s'imposent dans l'analyse.

Le premier problème est clairement celui de l'efficacité de l'outil industriel. Les laiteries de la période communiste étaient assez performantes mais demeuraient loin des références occidentales. Les nouveaux propriétaires procèdent en général à un audit (technique, humain et commercial) détaillé des affaires avant d'agir. Si l'usine est obsolète, elle est fermée mais on évite cette issue mal perçue par les autorités! En général donc, on modernise la chaîne de fabrication et on investit pour respecter les normes environnementales et sanitaires ce qui augmente considérablement l'investissement, le double souvent. Les spécialistes français (Chalon Mégard) ou suédois (Tetra Pak, Alfa Laval) du matériel de laiterie accompagnent alors les entreprises occidentales avec lesquelles ils ont l'habitude de travailler. Cette remise à niveau est d'autant plus nécessaire qu'au début des années 1990, l'environnement était difficile : on a oublié d'investir, du matériel a parfois disparu...

Une nouvelle phase de rationalisation de l'outil industriel intervient dans la seconde partie de la décennie qui s'achève, stimulée par la récente crise laitière, peut-être aussi par l'intégration européenne. Les groupes restructurent alors leur nouveau patrimoine en fermant ou en (se) revendant certaines affaires jugées peu rentables ou devenues non stratégiques, selon des pratiques guère différentes de celles en cours à l'Ouest. Dès lors, la restructuration fait mal : en Slovaquie, un bon tiers des sites industriels a fermé depuis 2005 ! Lactalis tire un trait sur les fromageries tchèques de Kruh et de Dřevěnice. Bel, de son côté, remet en vente en 2010 l'entreprise tchèque Jaromericka, acquise seulement deux ans plus tôt! Il avait déjà fermé l'usine slovaque de Tvrdošin, peu après l'avoir achetée. Quant à Bongrain, les produits frais n'entrant pas dans sa stratégie d'entreprise, il cède son site de Zvolen à Senoble. Le plus souvent, les groupes ferment les sites les moins compétitifs et concentrent la collecte sur les meilleures usines, comme Friesland qui renforce son site hongrois de Debrecen (2006).

De fait, les échecs sont nombreux, comme en Pologne d'où se retirent Friesland Coberco (1998), SODIAAL (2001), Nestlé (2003) et Campina (2004). Idem en Slovaquie d'où partent 
Friesland (2006), Parmalat et Danone. En fait, le faible coût de la main-d'œuvre ne suffit pas pour obtenir le succès. Ainsi, Danone se heurta rapidement à d'énormes problèmes qualitatifs, allant même jusqu'à devoir importer du lait de France pour faire tourner son usine de Wola ! En 1998, le groupe change de stratégie, engage un partenariat avec les producteurs et la société Provimi à qui il achète l'aliment pour le bétail qu'il rétrocède aux paysans (RLF, 2004)! Fruit de cet acharnement, Danone est désormais leader des produits frais en Pologne. Son investissement slovaque est en revanche un échec et le site de Trnava est à vendre... Quant à l'usine tchèque d'Opočno, elle passe successivement aux mains de l'Américain Heinz, puis du Franco-Néerlandais Nutricia, avant d'être revendue au groupe national Moravia Lacto.

Par ailleurs, en dépit de la faible « distance culturelle » qui existe entre l'Est et l'Ouest, il faut savoir s'adapter aux comportements locaux, à la faible productivité structurelle, aux emplois pas toujours justifiés, au chômage caché. Certes, les évolutions sont plus rapides ici que dans le secteur agricole, mais il s'agit bien là d'un chantier considérable pour des entreprises habituées à un autre environnement. Le défi est à vrai dire moins important qu'en ex-URSS ou dans les Balkans.

\section{$B$ - Les entreprises étrangères à la conquête des consommateurs}

Les étrangers qui s'installent en Europe médiane sont donc de grands groupes industriels qui ont une parfaite expérience du système capitaliste, de l'internationalisation et de la reprise d'entreprises.

Pour eux, l'accès à ces nouveaux marchés apparaît comme l'élément décisif. Certes vers 1990-1993, la crise économique avait été si sévère que la consommation de produits laitiers avait reculé de $20 \%$, mais le potentiel existe et le marché intérieur s'est d'ailleurs à nouveau développé avec les années. Ces trois pays sont parmi les plus avancés de l'ancien Bloc de l'Est et regroupent 53 millions de consommateurs (38 en Pologne, 5 en Slovaquie, 10 en Tchéquie) au pouvoir d'achat significatif. Surtout, ces marchés sont dynamiques et on se rend vite compte qu'ils sont promis à une forte croissance (phénomène de rattrapage), d'autant qu'il y a la perspective de l'intégration européenne et de ses aides financières.

30 En outre, on peut s'appuyer sur les circuits de distribution déjà installés (Jednota en Slovaquie...) et surtout sur le réseau des hyper et supermarchés qui se densifie rapidement et transpose ici le système de distribution occidental, la consommation de masse.

31 Les industriels de l'Ouest de l'Europe, experts dans l'analyse des marchés, savent s'adapter à ce nouveau contexte. Ils imposent tout d'abord leurs marques leaders (Danone, Président, Cœur de Lion, Caprice des Dieux, Activia Yoplait, La Vache qui Rit...). Leur objectif est d'être présents dans les rayons des grandes surfaces qui s'installent dans ces pays et qu'ils connaissent bien puisqu'il s'agit des mêmes enseignes qu'en Europe occidentale. Le consommateur trouve alors sans difficultés dans les capitales, puis dans les villes moyennes, toutes les grandes marques françaises que l'on vient de citer! Il peut acheter sans peine du camembert Président, des yaourts Activia, toute la gamme des produits Nestlé et même de nombreux fromages d'AOC français ou italiens. Ce processus classique de diffusion des marques concerne tant les produits frais que les fromages et s'impose auprès de consommateurs qui adoptent vite des comportements typiquement «occidentaux » rompant avec l'univers de pénurie et de 
médiocrité de l'offre des années communistes : attrait des marques, du packaging, rôle de la publicité...

La diffusion de ces marques relève exclusivement d'une stratégie commerciale, jamais de choix industriels conduits sur place. En clair, ces produits sont systématiquement importés. Pourquoi? En fait, ces fabrications très techniques sont parfaitement maitrisées dans les usines occidentales, sur des chaînes performantes où l'on a tout intérêt à augmenter les cadences, réalisant au passage des économies d'échelle. Par ailleurs, le consommateur d'Europe centrale est prêt à payer un bon prix ces marques renommées, ce qui permet de compenser les coûts d'approche. Peut-être aussi peut-on ainsi mieux protéger ses secrets de fabrication... Rien ne pousse les industriels occidentaux à délocaliser ces fabrications.

Ces mêmes linéaires offrent aussi une grande diversité de marques commerciales que l'on peut qualifier de «nationales ». Un peu moins chers, ces produits frais et fromages s'appuient sur des marques réputées (Niva pour un bleu tchèque, Hermelin pour une pâte molle du même pays, et, en Slovaquie, Rajo ou Agro milk pour les yaourts, Liptov pour toute une gamme de produits, Syrokrem pour du fromage à tartiner). Ces produits sont toujours d'une qualité certaine, tiennent la comparaison avec les articles étrangers et résistent, à l'évidence, nettement mieux que dans les Balkans ou en ex-URSS où les filières ont été déstructurées.

On rencontre aussi nombre de spécialités nationales, voire locales, que l'observateur français qualifierait de produits de terroir. Les fromages de type tvaroh, peu affinés et souvent consommés au petit déjeuner (twarog polonais), font partie de ces spécialités recherchées par les clients. Celles-ci sont omniprésentes en Slovaquie et dans les Carpates polonaises, à la faveur de l'expression de vrais savoir-faire fromagers montagnards : korbačiky, oštiepok (oscipek en Pologne), parenice et surtout bryndza. Les Tchèques, eux, par absence de tradition fromagère, ignorent ces produits si l'on excepte le réputé (mais très local) tvarošky Olomouc. Ces spécialités peuvent s'appuyer, à l'occasion, sur des marques commerciales (Liptov...) qui existaient souvent dès avant 1989.

Conséquence de cette attitude complexe des consommateurs, les usines des groupes étrangers sont essentiellement orientées vers quatre types de fabrications. Des produits à marques commerciales donc, des produits "de terroir ", surtout en Slovaquie, des produits frais (yaourts, lait UHT) et nombre de produits industriels génériques, assez basiques: pâtes pressées façon édam ou emmental, pâtes molles de type camembert, poudres, beurre...

Les orientations productives de ces usines n'ont donc pas été totalement bouleversées. En revanche, la restructuration des lignes de production de chaque site a été drastique, on l'a dit, marquée par la réduction de la gamme des produits, en rupture avec les logiques socialistes. Tel est le cas de l'usine de Liptovsky Mikulaš, qui produisait presque tout quand elle fut reprise par Bongrain. Cette rationalisation permet de gagner en efficacité et elle est amplifiée quand l'investisseur possède plusieurs usines dans le même pays. 


\section{IV - Les stratégies des firmes : quelles spécificités en Europe centrale?} largement reprises en Europe centrale : rationalisation de la collecte, amélioration de l'outil industriel, accroissement de la productivité, économies d'échelle, maîtrise sanitaire, certifications de type ISO... Ce processus de modernisation fonctionne plutôt bien dans des Etats certes différents de ceux de l'Europe occidentale, certes confrontés à des difficultés réelles, mais stables politiquement et qui appartiennent bien au même univers culturel que celui de l'Europe occidentale. Certaines spécificités intéressent plus particulièrement le géographe.

\section{A - La question sanitaire : pasteurisation ou lait cru ?}

Dans l'ensemble, dès le début de la transition, la maitrise de la qualité sanitaire du lait était correcte, à l'exception notable de la Pologne paysanne, largement en retard en la matière. L'arrivée des normes européennes, dès avant 2004 en réalité, n'a donc pas été un choc insurmontable, même s'il a fallu faire des efforts et la qualité sanitaire de la collecte est globalement satisfaisante, au moins en ex-Tchécoslovaquie. qui ont changé. La pasteurisation d'avant 1989 répond à une logique de croissance quantitative et de banalisation de l'alimentation socialiste. Aujourd'hui, c'est plutôt le rôle de la grande distribution et de l'Administration qui semble décisif. La première, soucieuse de ne pas avoir d'ennuis, favorise les produits pasteurisés. L'Administration, omniprésente et assez rigide, est toujours très hygiéniste... Elle favorise la pasteurisation... à tel point qu'on est souvent convaincu que Bruxelles « interdit le lait cru »! Il faut dire que la société locale privilégie plutôt la qualité sanitaire, comme en Allemagne. Les consommateurs apparaissent, à l'inverse, mal à l'aise face aux problématiques de qualité spécifique, de terroir. Certes, pour les Slovaques, la bryndza est une référence alimentaire, mais ils n'en consomment finalement que 5000 tonnes par an et surtout, la « vraie » bryndza, au lait cru de brebis, est devenue une rareté que l'on ne trouve plus guère que chez Manica à Tisovec, en Slovaquie centrale (Lompech, Ricard, 2007)!

\section{$B$ - Les territoires des firmes}

Partout, les IAA organisent leurs territoires et structurent des circuits de collecte qui sont le support essentiel de bassins de production, laitiers en l'occurrence. De tels phénomènes s'observent naturellement ici, avec toutefois des spécificités assez marquées.

Quand l'entreprise ne développe en Europe centrale que le négoce de produits fabriqués à l'Ouest, la notion de territoire des firmes n'existe guère. Le commerce peut aussi porter sur des produits industriels banalisés (lait en poudre écrémé, caséine, 
beurre en cube...) vendus à de gros acheteurs et il n'est alors jamais question de logiques territoriales. On peut aussi s'efforcer de placer directement auprès du consommateur des marques réputées, en privilégiant alors les relations commerciales avec les grandes surfaces. Le territoire de la firme se calque finalement plus ou moins sur celui de la grande distribution. Parfois enfin, on développe des franchises avec des partenaires locaux, comme SODIAAL pour sa marque Yoplait. Là encore les aspects territoriaux passent au second plan.

43 Le cas le plus intéressant reste celui de l'entreprise qui reprend à la fois l'usine et la collecte, deux éléments clefs de l'organisation spatiale des territoires par les firmes. Allons plus loin.

En Europe occidentale, où l'agriculture familiale domine, les sociétés cherchent à rationaliser le ramassage pour réduire les coûts de collecte. Elles tentent de densifier le cœur de zone et de marginaliser les producteurs périphériques, opération rendue difficile par l'existence de quotas souvent attachés au foncier ! En Europe centrale, la présence de grosses fermes héritées de l'ancien système change la donne, la Slovaquie détenant à ce titre le record européen de livraison de lait par exploitation (921 $000 \mathrm{l}$ en 2005). Le camion du laitier peut donc se permettre quelques détours pour récupérer une poignée d'exploitations livrant chacune deux ou trois millions de litres de lait! On remplit alors facilement une semi-remorque tous les deux jours et l'affaire est rentable, quitte à s'éloigner de 100 ou 150 kilomètres du quai de l'usine, comme la laiterie de Liptovsky Mikulaš qui collecte jusque dans le Spisš.

Le monde des petits producteurs des vieilles terres paysannes de Pologne orientale et centrale offre un autre type d'organisation territoriale. Ici, les problèmes sont nombreux pour les firmes qui doivent faire œuvre de vulgarisation technique auprès de petits agriculteurs mal équipés et peu performants sur le plan sanitaire, installer des tanks à lait, organiser et gérer la collecte, prévoir parfois des points de regroupement des bidons de lait. C'est un énorme investissement que Danone sut mener à bien, à la différence de la coopérative irlandaise Avonmore, arrivée dans le Sud du pays en 1993, mais qui capitula peu après.

L'investissement en Europe centrale mérite aussi d'être raisonné dans le cadre plus global de la stratégie de chaque groupe.

Quand ce dernier est bavarois ou autrichien, l'aire de ramassage peut être relativement proche de la maison mère et l'on peut procéder à des échanges de lait. Cela semble être le cas de Rajo à Bratislava, qui traite manifestement du lait d'importation, autrichien ou allemand. Autre cas de figure dans la Šumava où plusieurs grosses fermes, mécontentes de la laiterie Madeta de Česke Budejovice, ont récemment choisi de livrer leur lait en Bavière toute proche, à la faveur de prix du lait plus attractifs. L'aire de collecte de la firme allemande s'étend alors en tache d'huile par-delà la frontière.

Presque toujours toutefois, les firmes occidentales sont loin de leurs bases et la complémentarité des zones de ramassage disparaît. Le lait reste en effet un produit pondéreux et il n'est rentable, ni d'approvisionner les usines bretonnes ou même lorraines en lait polonais, ni de transformer du lait néerlandais ou belge en Europe centrale, sauf à la marge. La finalité première de ces usines est donc bien de satisfaire le marché local. 
49 A l'inverse, les Occidentaux peuvent se servir de ces usines comme d'une plateforme vers l'Est : avoir un atelier en Pologne permet d'explorer de nouveaux marchés, en Ukraine, en Russie, demain en Biélorussie.

\section{C - Des processus de délocalisation?}

La délocalisation industrielle anime les conversations, surtout en France. On ferme alors les usines pour transférer la production vers un autre lieu, offrant généralement de faibles coûts salariaux et la production diminue à l'Ouest pour augmenter à l'Est. La délocalisation peut aussi être différée, comme on l'observe dans l'industrie automobile : les nouvelles usines tchèques ou slovaques s'avèrent plus efficaces que celles de Sochaux ou de Flins et la production y augmente quand elle diminue en France... Dans le domaine laitier, de tels phénomènes ne s'observent qu'à la marge, et ce pour plusieurs raisons.

51 Premier point, la collecte laitière de ces trois pays ne progresse pas et a même tendance à diminuer en Slovaquie et en Tchéquie. Et si elle augmente légèrement en Pologne (Tab. 1), c'est uniquement par recul de l'autoconsommation au profit du ramassage. Il n'y a donc aucune délocalisation de la production de la matière première. A cela plusieurs raisons. Les quotas tout d'abord lient la production à la terre et l'on ne peut pas les transférer de Bretagne en Pologne, d'Espagne en Estonie. Il y a donc une forme de blocage administratif de la géographie de la production, qui devrait d'ailleurs sauter, au moins en partie, en 2015. Mais surtout, délocaliser la production «à l'Est» supposerait que ces pays offrent un vrai potentiel de développement. Or, l'analyse montre que les exploitations familiales modernisées de l'Europe occidentale sont sensiblement plus performantes que les anciennes fermes collectives d'Europe centrale, à l'exception peut-être des nouveaux länder.

D'autre part, il n'est pas rationnel pour les entreprises de transformation de produire du camembert en Slovaquie pour le rapatrier ensuite en France ou en Espagne, compte tenu, là encore, des coûts de transport. Et il n'y aurait pas plus de cohérence à ramener du lait UHT (un produit à faible marge) de Pologne à Paris. Le mouvement inverse de vente de produits occidentaux dans nos trois pays est éventuellement possible, mais surtout parce que certaines marques réputées (Président, Caprice des Dieux, Leerdamer...) autorisent un niveau de prix qui compense les frais d'approche. Au final, l'incidence des coûts de transport reste importante et le marché des produits laitiers conserve une base nationale particulièrement forte, à l'exception des petits pays, des zones frontalières et des produits industriels banalisés (lait en poudre...) dont le marché est très différent.

Résultat, les Hollandais ont bien fabriqué du gouda à Strážke, en Slovaquie orientale, mais essentiellement pour le marché local. Produire ici pour le marché néerlandais n'a en effet pas de sens, compte tenu de la distance et surtout de la compétitivité respective des usines de Strážke et des Pays-Bas. L'aventure a d'ailleurs tourné court, l'atelier a fermé, le lait va à Debrecen ... et les Slovaques mangent du gouda de Hollande!

Restent les Italiens d'Orrero qui exportent l'intégralité de leur production de Gran Moravia dans la Péninsule. S'agit-il d'une délocalisation pour un produit, le parmesan, au marché totalement internationalisé ? En tout cas, cette pâte dure vendue assez chère 
peut supporter le coût du transport, d'autant que LitoveL' n'est qu'à $800 \mathrm{~km}$ de la plaine du Pô.

$\mathrm{Au}$ final, les phénomènes de délocalisation restent donc marginaux, à cause notamment des coûts de transport, du rôle particulier des quotas et de la permanence de certaines habitudes alimentaires. Tout cela n'interdit pas une certaine internationalisation des filières, surtout à proximité des frontières. On a vu que des fermes tchèques livraient leur lait en Allemagne. Il peut aussi être rentable de transformer à Bratislava du lait collecté en Bavière ou en basse Autriche, peut-être même pour revendre les produits finis dans ces deux pays. De même, du lait UHT polonais inonde les linéaires allemands. Et d'ailleurs, le commerce extérieur de produits laitiers de ces trois pays ne cesse de se développer, signe de leur intégration croissante au sein de l'économie européenne.

\section{D - La question de la gouvernance des entreprises}

La gestion des laiteries d'Europe centrale par leurs nouveaux propriétaires est assez largement calquée sur leurs pratiques occidentales. Les différences dans la gouvernance des sociétés sont alors importantes et se retrouvent dans la gestion de ce nouveau patrimoine.

57 La plupart des entreprises laitières d'Europe de l'Ouest sont réputées pour être très centralisées et la prise de décisions a alors systématiquement lieu en France, en Allemagne ou aux Pays-Bas, au siège du groupe, avant application sur place par une équipe dirigeante qui compte nombre de cadres expatriés. Mais d'autres sociétés sont structurellement beaucoup plus décentralisées, à l'image du Français Bongrain. La visite de son usine de Liptovský Mikulaš est à cet égard très éclairante. Ici, dans la plus grosse fromagerie du pays, le management est exclusivement slovaque et la secrétaire de direction assure seule la traduction et donc le lien avec la France. Une grande autonomie est donc laissée à l'échelon local.

\section{Conclusion}

La transformation laitière représente un pan important de l'industrie nationale en Slovaquie, en République Tchèque et en Pologne, même si l'on reste loin de la puissance des industries néerlandaises ou danoises, voire bretonnes. Ce secteur d'activité, déjà actif avant 1989, a connu de profonds bouleversements avec la transition postcommuniste et est rapidement passé aux mains des étrangers qui disposent désormais ici des parts de marché très importantes. Leurs modalités d'implantation ont toutefois été très variées, avec toute une géographie des IDE qui s'est progressivement dessinée, au profit notamment de la France.

Vingt ans après, le bilan est manifestement positif. Les usines ont été modernisées, leur impact environnemental a été fortement réduit, la productivité s'est sensiblement améliorée et les aspects sanitaires sont bien maîtrisés. Cette dynamique de progrès est incontestablement plus marquée qu'au stade de la production laitière où les anciennes fermes collectives n'ont pas tenu leurs promesses et où les nouveaux producteurs individuels trouvent difficilement leur place. Tout ce mouvement d'internalisation des entreprises montre des spécificités réelles par rapport au reste de l'industrie, les phénomènes de délocalisation notamment n'apparaissant pas comme une préoccupation majeure dans la stratégie des firmes venues investir ici. 


\section{BIBLIOGRAPHIE}

Blaha J., Borzeda A., 1999, L'industrie des boissons alcoolisées en Europe centrale et orientale, Le Courrier des Pays de l'Est, $n^{\circ} 441$, p. 26-47.

Cahiers de l'ONILAIT, $\mathrm{n}^{\circ}$ 7, juillet 1992, « Pologne, Hongrie, Tchécoslovaquie, la filière laitière ", Paris, $50 \mathrm{p}$.

Carlier J.-P., 2000, Le secteur laitier polonais à la veille de son intégration dans l'Union Européenne, Rapport de mission d'étude pour ATLA, $64 \mathrm{p}$.

Carlier J.-P., 2001, Le secteur laitier slovaque à la veille de son intégration dans l'Union Européenne, Rapport de mission d'étude pour ATLA, $108 \mathrm{p}$.

Domansky B., 2001, Kapitał zagraniczny w przemyśle Polski, Krakow, 522 p.

Dries L., 2004, Vertical coordination and Foreign Direct Investment. A comparative study of the dairy chains in Bulgaria, Poland and Slovakia, Rapport pour la Banque Mondiale, $34 \mathrm{p}$.

Groupes Lactalis, Bel, Bongrain, Entremont, SODIAAL, Arla Foods, Humana, Nordmilch, Hochland, Meggle, Ehrmann, BMI, Müller, Nestlé, Valio, Friesland Campina, Parmalat, rapports annuels et sites Internet.

Institut de l'Elevage, 2007, La filière laitière en Pologne. Un fort potentiel, Dossier Economie de l'Elevage, $\mathrm{n}^{\circ} 370$.

Lacquement G., 1996, La décollectivisation dans les nouveaux Länder : acteurs et territoires face au changement de modèle agricole, Paris, L'Harmattan, 225 p.

Lompech M., 2004, Un bassin de production agricole en transformation : les agro-industries de Rimavská Sobota, Dynamiques agro-industrielles et dynamiques rurales, Arras, 242 p., p. 65-88.

Lompech M., Ricard D., 2007, Un produit spécifique de la montagne slovaque : la bryndza face à l'intégration européenne, in Actes du colloque de Vallouise, L'alimentation en montagne, p. 119-130.

Maciulyte J., 2001, Les mutations agraires de l'espace lituanien, Territoires en mutation, Montpellier, $\mathrm{n}^{\circ} 8,186 \mathrm{p}$.

Potravinářský Zpravodaj, revue professionnelle tchèque de l'agroalimentaire, Prague.

Pouliquen A., 1991, D'un système à l'autre. La situation du secteur agroalimentaire en Tchécoslovaquie, Cahiers d'Economie et de Sociologie Rurale $\mathrm{n}^{\circ}$ 21, p. 45-69.

Pouliquen A., 1997, L'illusoire compétitivité agricole des PECO : vers un élargissement des exportations de l'Union européenne,Le Courrier des Pays de l'Est, nº 416, p. 51-56.

Pouliquen A., 2001, Compétitivité et revenus agricoles dans les secteurs agroalimentaires des PECO. Implications avant et après l'adhésion pour les marchés et les politiques de l'Union Européenne, Rapport pour la Commission Européenne, $72 \mathrm{p}$.

Revue Laitière Française, revue professionnelle mensuelle, Caen.

Simon F., 1994, La transition dans le secteur agroalimentaire tchèque : de la propriété publique à la propriété privée,Economie Rurale, $\mathrm{n}^{\circ} 223$, p. 46-52.

Simon F., 1995, De l'appropriation juridique à l'appropriation économique : le rôle des acteurs. L'exemple de la privatisation dans le secteur agroalimentaire tchèque, thèse de doctorat ENSA, Montpellier, 474 p. 
Šimunek J., Regionálne prístupy k rozvoju mliekarenského priemyslu na Slovensku, Ekonomika pol'nohospodárstva, VIII, 2008, č. 3, p. 29-32.

Spissiak P., 1995, Agrosystém na Slovensku po roku 1989, in KOWALCZYK A. (Ed.), Zmiany w przestrzeni geograficznej $w$ warunkach transformacji spoleczno-ekonomiczenej na przkyladzie obszarów, coll. « Wydzial Geografii i Studiów Regionalnich », Uniwersytet Warszawski, Warsovie, p. 176-181.

Sznajder M., Krusinska O., Wielicka A., 2005, Review of dairy sector in Poland, Roczniki Akademii Rolniczej w Poznaniu, CCCLXVII, Université d'Agriculture de Poznań.

Ubifrance, Le secteur laitier en République Tchèque, note de synthèse, 4 p., février 2008, Mission économique Prague.

Ubreziova I., 2005, Internationalization process and changes in Slovak milk industry / Procesu internacionalizácie a zmeny $v$ slovenskom mliekarenskom priemysle, Agric. Econ. Czech, 51, (8), p. 357-361.

Von Hirschhausen B., Les nouvelles campagnes roumaines. Paradoxe d'un « retour paysan ", Coll. Mappemonde, Belin, Paris, 240 p.

Vuepp, 1999, Analýza vývoja poL’nohospodarstva a potravinárstva v období rokov 1990-1998, Bratislava, $184 \mathrm{p}$.

Zrinscak G., 1997, Mutations des campagnes tchèques, une décollectivisation pragmatique, Coll. Mappemonde, Belin, Paris, 256 p.

\section{RÉSUMÉS}

La transition post communiste a bouleversé l'industrie de l'ancien bloc de l'Est. L'industrie laitière s'inscrit dans ce mouvement, marquée notamment par l'arrivée massive d'investisseurs étrangers venus d'Europe de l'Ouest, notamment de France. Ces derniers ont largement réorganisé l'outil industriel laitier des trois pays étudiés (Pologne, Slovaquie, Tchéquie), le rendant nettement plus efficace sans que l'on observe de processus de délocalisation, ni de la production du lait, ni de la transformation, sauf à la marge. L'article décrit ce mouvement de conquête et en souligne les spécificités : profil des investisseurs, type d'investissements, rôle des marques, question sanitaire, relations entre les firmes et leur territoire...

Post communist transition has disrupted the industry of the former Eastern bloc. Milk industry is in keeping with this movement, and notably marked by a massive arrival of foreign investors coming from Western Europe, mainly France. They have greatly reorganized the dairy industrial tool of the three studied countries (Poland, Slovakia and the Czech Republic), making it distinctly more effective without relocating either milk production, or transformation, except for very few exceptions. The article depicts this conquest and underlines its specificities: investors' profile, type of investments, role of brand names, sanitary issues, relations between firms and their territory...

Die post-sozialistische Transformation hat im ehemaligen Ostblock die Agrarindustrie verwandelt. Die Entwicklungen in der Milchindustrie sind unter anderem durch die massiven Investitionen aus dem westeuropäischen Ausland, insbesondere aus Frankreich, gekennzeichnet. In Polen, der Slowakei und der tschechischen Republik haben diese ausländischen Investoren die Milchindustrie restrukturiert und effizienter gemacht, jedoch fast ohne Delokalisierung der Milchproduktion und -transformation. Der Artikel beschreibt diese räumlich-wirtschaftlichen 
Entwicklungen und ihre Besonderheiten: Bild der Investoren, Investitionstyp, Rolle der Qualitätszeichen, sanitäre Frage, Raum-Unternehmen-Verhältnis...

\section{INDEX}

Schlüsselwörter : Agro Industrie, Central Europa, Milch, Polen, post-sozialistische Transformation, Slowakei, Tschechischen Republik

Mots-clés : Europe centrale, firmes, industrie agroalimentaire, lait, Pologne, République tchèque, Slovaquie, transition post communiste

Keywords : central Europe, Czech Republic, firms, food processing industry, milk, Poland, post communist transition, Slovakia

\section{AUTEUR}

\section{DANIEL RICARD}

Clermont Université, Université Blaise-Pascal, EA 997, Ceramac, MSH, 4 rue Ledru, 63057

Clermont-Ferrand Cedex 1 